\title{
Algebras of Toeplitz operators with oscillating symbols
}

\author{
Albrecht Böttcher, Sergei M. Grudsky \\ and Enrique Ramírez de Arellano
}

\begin{abstract}
This paper is devoted to Banach algebras generated by Toeplitz operators with strongly oscillating symbols, that is, with symbols of the form $b\left(e^{i \alpha(x)}\right)$ where $b$ belongs to some algebra of functions on the unit circle and $\alpha$ is a fixed orientation-preserving homeomorphism of the real line onto itself. We prove the existence of certain interesting homomorphisms and establish conditions for the normal solvability, Fredholmness, and invertibility of operators in these algebras.
\end{abstract}

\section{Introduction and main results}

The Hardy space $H^{2}(\mathbb{R})$ is the closed subspace of $L^{2}(\mathbb{R})$ constituted by the functions $f$ of the form

$$
f(x)=\int_{0}^{\infty} g(t) e^{i t x} d t \quad(x \in \mathbb{R})
$$

with $g \in L^{2}(0, \infty)$. Let $L^{\infty}(\mathbb{R})$ be the $C^{*}$-algebra of all essentially bounded functions on $\mathbb{R}$. For $a \in L^{\infty}(\mathbb{R})$, the Toeplitz operator $T(a)$ is the bounded linear operator on $H^{2}(\mathbb{R})$ defined by $T(a) f=P(a f)$, where $P$ denotes the orthogonal projection of $L^{2}(\mathbb{R})$ onto $H^{2}(\mathbb{R})$. The function $a$ is referred to as the symbol of the operator $T(a)$.

Given a closed subalgebra $S$ of $L^{\infty}(\mathbb{R})$, we denote by $\mathcal{B}_{S}$ the Banach algebra generated by all Toeplitz operators with symbols in $S$. Thus, $\mathcal{B}_{S}$ is

2000 Mathematics Subject Classification: Primary 47B35; Secondary 30E20, 37C05, 42A50, 46H20, 47A53, 47L15.

Keywords: Toeplitz operator, Banach algebra, $C^{*}$-algebra, Fredholm operator, normally solvable operator. 
the closure in $\mathcal{L}\left(H^{2}(\mathbb{R})\right)$ of the set $\mathcal{B}_{S}^{0}$ of all operators of the form

$$
A=\sum_{j} \prod_{k} T\left(a_{j k}\right), \quad a_{j k} \in S
$$

the sum and the products finite. Such algebras have been extensively studied for many decades. We here confine ourselves to quoting the pioneering work [9], [16] for $S=C(\dot{\mathbb{R}})$ (the continuous functions on the one-point compactification of $\mathbb{R}$ ), [18], [19] for $S=P C(\mathbb{R})$ (the $C^{*}$-algebra of all piecewise continuous functions on $\mathbb{R}$ ), [11], [12] for $S=C(\dot{\mathbb{R}})+H^{\infty}(\mathbb{R})$ (the Douglas algebra), and $[29]$ for $S=P Q C(\mathbb{R})$ (the piecewise quasicontinuous functions on $\mathbb{R}$ ). The books [7] and [25] contain all these results together with full proofs. The key to the investigation of the algebras listed above is the happy circumstance that the commutator $T(a) T(b)-T(b) T(a)$ or even the semi-commutator $T(a) T(b)-T(a b)$ is compact for $a, b \in S$.

This paper is concerned with the algebras $\mathcal{B}_{S}$ in the case where $S$ is an algebra of strongly oscillating functions. For instance, we consider the case where $S$ is the algebra of all continuous $2 \pi / \lambda$-periodic functions on $\mathbb{R}$, that is, the algebra of all functions of the form $b\left(e^{i \lambda x}\right)$ with $b$ a continuous function on the complex unit circle. In the case of strongly oscillating symbols, the commutator $T(a) T(b)-T(b) T(a)$ is in general not compact. Algebras of Toeplitz operators with strongly oscillating symbols arise, for example, in the inverse scattering method for the modified Korteweg-de Vries equation (see, e.g., [2], [10], [24]). To state precise results, we need some more notation.

Let $H^{2}(\mathbb{T})$ be the Hardy space of the complex unit circle $\mathbb{T}$, that is, the space of all functions $f \in L^{2}(\mathbb{T})$ whose Fourier series is of the form

$$
f(t)=\sum_{k=0}^{\infty} f_{k} t^{k} \quad(t \in \mathbb{T})
$$

and let $P_{\mathbb{T}}$ be the orthogonal projection of $L^{2}(\mathbb{T})$ onto $H^{2}(\mathbb{T})$. It will be convenient to define the norm in $L^{2}(\mathbb{T})$ and $H^{2}(\mathbb{T})$ by

$$
\|f\|_{2}^{2}=\frac{1}{2 \pi} \int_{0}^{2 \pi}\left|f\left(e^{i \theta}\right)\right|^{2} d \theta .
$$

The Toeplitz operator on $H^{2}(\mathbb{T})$ generated by a function $b \in L^{\infty}(\mathbb{T})$ (its symbol) is the operator $T(b): H^{2}(\mathbb{T}) \rightarrow H^{2}(\mathbb{T}), f \mapsto P_{\mathbb{T}}(b f)$. For a closed subalgebra $R$ of $L^{\infty}(\mathbb{T})$, we define $\mathcal{B}_{R}$ as the closure in $\mathcal{L}\left(H^{2}(\mathbb{T})\right)$ of the set $\mathcal{B}_{R}^{0}$ of all operators of the form

$$
B=\sum_{j} \prod_{k} T\left(b_{j k}\right), \quad b_{j k} \in R,
$$

where the sum and the products are finite. 
Let $\alpha: \mathbb{R} \rightarrow \mathbb{R}$ be a homeomorphism that preserves the orientation of $\mathbb{R}$. Then $e^{i \alpha}$ is a map of $\mathbb{R}$ onto $\mathbb{T}$. Thus, if $b$ is a function on $\mathbb{T}$, then the function $b \circ e^{i \alpha}$ defined by $\left(b \circ e^{i \alpha}\right)(x)=b\left(e^{i \alpha(x)}\right)$ is a function living on $\mathbb{R}$. Note that typically $b \circ e^{i \alpha}$ is strongly oscillating.

Given a closed subalgebra $R$ of $L^{\infty}(\mathbb{T})$, we let $\mathcal{B}_{R}\left(e^{i \alpha}\right)$ denote the closure in $\mathcal{L}\left(H^{2}(\mathbb{R})\right)$ of the set $\mathcal{B}_{R}^{0}\left(e^{i \alpha}\right)$ of all operators that can be written as

$$
A=\sum_{j} \prod_{k} T\left(b_{j k} \circ e^{i \alpha}\right), \quad b_{j k} \in R,
$$

the sum and the products again finite. Equivalently, $\mathcal{B}_{R}\left(e^{i \alpha}\right)=\mathcal{B}_{S}$ where $S=\left\{b \circ e^{i \alpha}: b \in R\right\}$. It is algebras of the form $\mathcal{B}_{R}\left(e^{i \alpha}\right)$ that are the subject of this paper.

We remark that the map

$$
U: H^{2}(\mathbb{T}) \rightarrow H^{2}(\mathbb{R}), \quad(U f)(x)=\frac{1}{\sqrt{\pi}} \cdot \frac{1}{x+i} f\left(\frac{x-i}{x+i}\right)
$$

is an isometric isomorphism. If $b \in L^{\infty}(\mathbb{T})$, then

$$
U T(b) U^{-1}=T\left(b^{\#}\right),
$$

where $b^{\#} \in L^{\infty}(\mathbb{R})$ is given by

$$
b^{\#}(x)=a((x-i)(x+i)) .
$$

In this way all the results we will establish for Toeplitz operators on $H^{2}(\mathbb{R})$ can be easily transferred to Toeplitz operators on $H^{2}(\mathbb{T})$. We prefer the $H^{2}(\mathbb{R})$ setting because the line is more convenient than the circle when working with oscillating functions.

Let $H^{\infty}(\mathbb{R})$ be the Banach algebra of all functions in $L^{\infty}(\mathbb{R})$ that can be continued to bounded analytic functions in the upper half-plane $\mathbb{C}_{+}$. If $e^{i \alpha} \in H^{\infty}(\mathbb{R})$, then $e^{i \alpha}$ is an inner function, and we write $e^{i \alpha}=u$ in this case. Individual operators of the form $T(b \circ u)$ were studied in [3], [4], [13], [22], [25], for example. In particular, it is known that if $b \in L^{\infty}(\mathbb{T})$, then $T(b \circ u)$ is invertible if and only if $T(b)$ is invertible. One purpose of this paper is to extend results of this type to operators in $\mathcal{B}_{L^{\infty}(\mathbb{T})}(u)$. This will be done with the help of the following theorem.

Theorem 1.1 The map

$$
F_{u}: \mathcal{B}_{L^{\infty}(\mathbb{T})}^{0} \rightarrow \mathcal{B}_{L^{\infty}(\mathbb{T})}^{0}(u), \quad \sum_{j} \prod_{k} T\left(b_{j k}\right) \mapsto \sum_{j} \prod_{k} T\left(b_{j k} \circ u\right)
$$

is well-defined and extends to an (isometric) $C^{*}$-algebra isomorphism $F_{u}$ of $\mathcal{B}_{L^{\infty}(\mathbb{T})}$ onto $\mathcal{B}_{L^{\infty}(\mathbb{T})}(u)$.

The proof of this theorem will be based on the so-called "functional model" (see, e.g., [25]). 
Given a Hilbert space $H$, we denote by $\mathcal{L}(H)$ and $\mathcal{K}(H)$ the bounded and compact linear operators on $H$, respectively. An operator $T \in \mathcal{L}(H)$ is said to be normally solvable if its range $\operatorname{Im} T:=\{T h: h \in H\}$ is closed, and it is called Fredholm, left Fredholm, right Fredholm if the coset $T+\mathcal{K}(H)$ is invertible, left invertible, right invertible in the quotient algebra $\mathcal{L}(H) / \mathcal{K}(H)$. The kernel $\operatorname{Ker} T$ is defined as $\{h \in H: T h=0\}$. It is well known (see, e.g., [19]) that $T$ is left Fredholm if and only if $\operatorname{Im} T$ is closed and $\operatorname{dim} \operatorname{Ker} T<\infty$ and that $T$ is right Fredholm if and only if $\operatorname{Im} T$ is closed and $\operatorname{dim}$ Coker $T<\infty$, where Coker $T:=H / \operatorname{Im} T$. We here prove the following.

Theorem 1.2 If $A$ is in $\mathcal{B}_{L^{\infty}(\mathbb{T})}(u)$ and $B \in \mathcal{B}_{L^{\infty}(\mathbb{T})}$ is given by $A=F_{u}(B)$, then

$A$ is normally solvable $\Longleftrightarrow B$ is normally solvable,

$A$ is (left/right) Fredholm $\Longleftrightarrow A$ is (left/right) invertible,

$A$ is (left/right) invertible $\Longleftrightarrow B$ is (left/right) invertible.

Things are more complicated if $e^{i \alpha}$ is not an inner function. Our basic assumption on the homeomorphism $\alpha$ will be that

$$
e^{i \alpha} \in C(\dot{\mathbb{R}})+H^{\infty}(\mathbb{R}) .
$$

In [5] and [21], it was shown that this assumption is satisfied by many of interesting homeomorphisms $\alpha$ (see Section 3). Here is the main result of this paper.

Theorem 1.3 If (1.2) holds, then the map

$$
G_{\alpha}: \mathcal{B}_{C(\mathbb{T})}^{0}\left(e^{i \alpha}\right) \rightarrow \mathcal{B}_{C(\mathbb{T})}^{0}, \quad \sum_{j} \prod_{k} T\left(b_{j k} \circ e^{i \alpha}\right) \mapsto \sum_{j} \prod_{k} T\left(b_{j k}\right)
$$

is well-defined and extends to a (contractive) $C^{*}$-algebra homomorphism $G_{\alpha}$ of $\mathcal{B}_{C(\mathbb{T})}\left(e^{i \alpha}\right)$ onto $\mathcal{B}_{C(\mathbb{T})}$. The homomorphism $G_{\alpha}$ in injective and hence an (isometric) $C^{*}$-algebra isomorphism if and only if $e^{i \alpha} \in H^{\infty}(\mathbb{R})$.

The proof of this theorem makes use of the techniques developed in [20] and [21] (also see [3], [5], [6], [13]), where individual operators of the form $T\left(b \circ e^{i \alpha}\right)$ were considered, and the main ingredient is the observation that $e^{i \alpha}$ admits the factorization $e^{i \alpha}=u_{\alpha} c$ where $u_{\alpha}$ is an inner function and $c$ is a function in $C(\dot{\mathbb{R}})$ such that $c(\infty)=1$. 
It is well known that the map

$$
\operatorname{Sym}: \mathcal{B}_{L^{\infty}(\mathbb{R})}^{0} \rightarrow L^{\infty}(\mathbb{R}), \quad \sum_{j} \prod_{k} T\left(a_{j k}\right) \mapsto \sum_{j} \prod_{k} a_{j k}
$$

can be extended to a well-defined (contractive) $C^{*}$-algebra homomorphism of $\mathcal{B}_{L^{\infty}(\mathbb{R})}$ onto $L^{\infty}(\mathbb{R})$ (see [1], [7, Proposition 4.4], [11], [27] for $H^{2}(\mathbb{T})$ and use $(1.1)$ to pass to $\left.H^{2}(\mathbb{R})\right)$. The following theorem uncovers the structure of operators in $\mathcal{B}_{C(\mathbb{T})}\left(e^{i \alpha}\right)$.

Theorem 1.4 Suppose (1.2) holds and let $e^{i \alpha}=u_{\alpha} c$, where $u_{\alpha}$ is inner, $c \in C(\dot{\mathbb{R}}), c(\infty)=1$. Every operator $A \in \mathcal{B}_{C(\mathbb{T})}\left(e^{i \alpha}\right)$ is of the form

$$
A=F_{u_{\alpha}}(B)+T\left(\operatorname{Sym} A-\operatorname{Sym} F_{u_{\alpha}}(B)\right)+K
$$

where $B=G_{\alpha}(A)$ and $K$ is a compact operator.

Here is an analogue of Theorem 1.2 in the case where $e^{i \alpha}$ is not required to be analytic.

Theorem 1.5 Let (1.2) be satisfied. If $A \in \mathcal{B}_{C(\mathbb{T})}\left(e^{i \alpha}\right)$ and $B=G_{\alpha}(A)$, then

$A$ is normally solvable $\Longrightarrow B$ is normally solvable,

$A$ is (left/right) Fredholm $\Longleftrightarrow B$ is (left/right) invertible.

This theorem can be proved by localization techniques: note that at infinity the operator $\sum_{j} \prod_{k} T\left(b_{j k} \circ e^{i \alpha}\right)$ is locally equivalent to the operator $\sum_{j} \prod_{k} T\left(b_{j k} \circ u_{\alpha}\right)$ and thus to an operator as in Theorem 1.2. We will proceed differently and derive Theorem 1.5 from Theorem 1.4.

If $e^{i \alpha}=u \in H^{\infty}(\mathbb{R})$, then Theorem 1.2 shows that in the assertion of Theorem 1.5 which concerns normal solvability the implication " $\Longrightarrow$ " can even be replaced by the equivalence " $\Longleftrightarrow$ ". As the following result reveals, this is no longer true if $e^{i \alpha}$ is not analytic.

Theorem 1.6 Let $e^{i \alpha}$ be in $C(\dot{\mathbb{R}})+H^{\infty}(\mathbb{R})$ but not in $H^{\infty}(\mathbb{R})$. Then there exist operators $A \in \mathcal{B}_{C(\mathbb{T})}\left(e^{i \alpha}\right)$ such that $A$ is not normally solvalble although $B=G_{\alpha}(A)$ is normally solvable.

The paper is organized as follows. Section 2 deals with algebras generated by Toeplitz operators of the form $T(b \circ u)$ where $u$ is an arbitrary inner function. In the special case where $u=e^{i \alpha}$ with an orientation-preserving homeomorphism $\alpha: \mathbb{R} \rightarrow \mathbb{R}$, these results imply Theorems 1.1 and 1.2. Section 3 is devoted to the proof of Theorem 1.3, and in Section 4 we prove Theorems 1.4 to 1.6 .

We remark that our proofs show that Theorems 1.1 to 1.6 remain literally true in the matrix case, that is, for algebras generated by operators of the form $T\left(b \circ e^{i \alpha}\right)$ where $b$ is a matrix function on $\mathbb{T}$ and $\alpha$ is a fixed homeomorphism of $\mathbb{R}$ onto itself that preserves the orientation of $\mathbb{R}$. 


\section{Oscillations caused by inner functions}

The results of this section will imply Theorems 1.1 and 1.2.

Let $u \in H^{\infty}(\mathbb{R})$ be an inner function. We do not assume that $u$ is of the form $e^{i \alpha}$ with an orientation-preserving homeomorphism $\alpha: \mathbb{R} \rightarrow \mathbb{R}$. However, we assume that $u$ is not a finite Blaschke product (this case will be treated separately in the end of this section).

The set $u H^{2}(\mathbb{R}):=\left\{u h: h \in H^{2}(\mathbb{R})\right\}$ is a closed subspace of $H^{2}(\mathbb{R})$. Let $K_{u}$ denote the orthogonal complement of $u H^{2}(\mathbb{R})$ in $H^{2}(\mathbb{R})$ :

$$
H^{2}(\mathbb{R})=u H^{2}(\mathbb{R}) \oplus K_{u} .
$$

It is easily seen that

$$
K_{u}=\operatorname{Ker} T(\bar{u}),
$$

and since $u$ is not a finite Blaschke product, $\operatorname{dim} K_{u}=\infty$ (see, e.g., [7, Theorem 2.64] or [25]). The following lemma is a well known consequence of the Wold decomposition (see, e.g., [23], [25]). We include an elementary proof for the reader's convenience.

Lemma 2.1 We have

$$
H^{2}(\mathbb{R})=\bigoplus_{j=1}^{\infty} K_{u}^{(j)} \quad \text { with } \quad K_{u}^{(j)}:=u^{j} K_{u}
$$

Proof. If $f, g \in K_{u}$ and $j<k$, then

$$
\left(u^{j} f, u^{k} g\right)=\left(\bar{u}^{k-j} f, g\right)=\left(\bar{u}^{k-j} f, P g\right)=\left(P\left(\bar{u}^{k-j} f, g\right)=\left(T(\bar{u})^{k-j} f, g\right),\right.
$$

and this is zero by virtue of (2.2). Thus, it remains to show that $\oplus_{j=1}^{\infty} K_{u}^{(j)}$ is dense in $H^{2}(\mathbb{R})$. Assume the contrary: there exists a $g \in H^{2}(\mathbb{R})$ such that $g \neq 0$ and $g \perp K_{u}^{(j)}$ for all $j$. It follows that $\left(\bar{u}^{j} g, h\right)=\left(g, u^{j} h\right)=0$ for all $h \in K_{u}$ and all $j$, whence $\bar{u}^{j} g \in u H^{2}(\mathbb{R})$ for all $j$ due to (2.1). Consequently, $g \in u^{j+1} H^{2}(\mathbb{R})$ for all $j$. Thus, $g=u^{j+1} g_{j}$ with $g_{j} \in H^{2}(\mathbb{R})$ for all $j$. Since $g \neq 0$, there is a point $z_{0}$ in the upper half-plane such that $g\left(z_{0}\right) \neq 0$. As $\left|g\left(z_{0}\right)\right|=\left|u\left(z_{0}\right)\right|^{j+1}\left|g_{j}\left(z_{0}\right)\right|$ and $\left|u\left(z_{0}\right)\right|<1$, we conclude that $\left|g_{j}\left(z_{0}\right)\right| \rightarrow \infty$ as $j \rightarrow \infty$. But Cauchy's integral formula tells us that

$$
g_{j}\left(z_{0}\right)=\frac{1}{2 \pi i} \int_{\mathbb{R}} \frac{g_{j}(x)}{x-z_{0}} d x
$$

and the Cauchy-Schwarz inequality therefore gives

$$
\left|g_{j}\left(z_{0}\right)\right| \leq \frac{\left\|g_{j}\right\|_{2}}{2 \pi}\left(\int_{\mathbb{R}} \frac{d x}{\left|x-z_{0}\right|^{2}}\right)^{1 / 2}=\frac{\|g\|_{2}}{2 \pi}\left(\int_{\mathbb{R}} \frac{d x}{\left|x-z_{0}\right|^{2}}\right)^{1 / 2} .
$$

As the integral in the last estimate is finite, we have a contradiction. 
From the theory of composition operators it is well known that if $f \in H^{2}(\mathbb{T})$ and $u$ is an inner function, then the function $f(u(x)) /(x+i)$ belongs to $H^{2}(\mathbb{R})$ (see, e.g., [26], [30]). Clearly, $f \circ u$ itself need not be in $H^{2}(\mathbb{R})$ (take, for example, $f=1$ ). However, if we multiply $f \circ u$ by a function in $K_{u}$, then the result is always in $H^{2}(\mathbb{R})$. This is the content of the following lemma.

Lemma 2.2 Let $e \in K_{u}$ and $\|e\|_{2}=1$.

(a) If $f \in L^{2}(\mathbb{T})$ and $k \in \mathbb{Z}$, then $e u^{k}(f \circ u) \in L^{2}(\mathbb{R})$ and $\left\|e u^{k}(f \circ u)\right\|_{2}=$ $\|f\|_{2}$.

(b) If $f \in H^{2}(\mathbb{T})$, then $e(f \circ u) \in H^{2}(\mathbb{R})$ and $\|e(f \circ u)\|_{2}=\|f\|_{2}$.

Proof. This follows from the equalities

$$
\begin{aligned}
\left\|e u^{k}(f \circ u)\right\|_{2}^{2} & =\left(e u^{k} \sum f_{j} u^{j}, e u^{k} \sum f_{l} u^{l}\right) \\
& =\sum_{j, l} f_{j} \bar{f}_{l}\left(e u^{k+j}, e u^{k+l}\right) \\
& =\sum_{j}\left|f_{j}\right|^{2}(e, e) \quad(\text { Lemma 2.1) } \\
& =\sum_{j}\left|f_{j}\right|^{2}=\|f\|_{2}^{2} .
\end{aligned}
$$

The following lemma describes the action of $T(b \circ u)$ on functions of the form $e(f \circ u)$.

Lemma 2.3 Let $e \in K_{u}, f \in H^{2}(\mathbb{T}), b \in L^{2}(\mathbb{T})$. Then

$$
T(b \circ u)(e(f \circ u))=e(T(b) f \circ u) .
$$

Proof. Define $\chi_{n}$ by $\chi_{n}(t)=t^{n}(t \in \mathbb{T})$. For $j \in \mathbb{Z}$ and $k \in \mathbb{Z}_{+}$,

$$
T\left(\chi_{j} \circ u\right)\left(e\left(\chi_{k} \circ u\right)\right)=P\left(u^{j} e u^{k}\right)=\left\{\begin{array}{cl}
e u^{j+k} & \text { if } j+k \geq 0, \\
0 & \text { if } j+k<0,
\end{array}\right.
$$

and

$$
e\left(T\left(\chi_{j}\right) \chi_{k} \circ u\right)=e\left(P\left(\chi_{j+k}\right) \circ u\right)=\left\{\begin{array}{cl}
e u^{j+k} & \text { if } j+k \geq 0, \\
0 & \text { if } j+k<0 .
\end{array}\right.
$$

This implies (2.3) for $b=\chi_{j}$ and $f=\chi_{k}$. From Lemma 2.2(a) we deduce that if $b \in L^{2}(\mathbb{T})$, then

$$
\begin{aligned}
\left\|(b \circ u)\left(e\left(\chi_{k} \circ u\right)\right)\right\|_{2} & =\left\|e u^{k}(b \circ u)\right\|_{2}=\|b\|_{2}, \\
\left\|e\left(T(b) \chi_{k} \circ u\right)\right\|_{2} & =\left\|T(b) \chi_{k}\right\|_{2} \leq\|b\|_{2},
\end{aligned}
$$

which shows that the maps

$$
b \mapsto T(b \circ u)\left(e\left(\chi_{k} \circ u\right)\right), \quad b \mapsto e\left(T(b) \chi_{k} \circ u\right)
$$

are bounded linear operators on $L^{2}(\mathbb{T})$. 
Consequently, (2.3) is true for all $b \in L^{\infty}(\mathbb{T}) \subset L^{2}(\mathbb{T})$ and for $f=\chi_{k}$. Finally, for $b \in L^{\infty}(\mathbb{T})$ and $f \in H^{2}(\mathbb{T})$ we obtain from Lemma 2.2(b) that

$$
\begin{aligned}
\|T(b \circ u)(e(f \circ u))\|_{2} & \leq\|b\|_{\infty}\|e(f \circ u)\|_{2}=\|b\|_{\infty}\|f\|_{2}, \\
\|e(T(b) f \circ u)\|_{2} & =\|T(b) f\|_{2} \leq\|b\|_{\infty}\|f\|_{2}
\end{aligned}
$$

and hence the maps

$$
f \mapsto T(b \circ u)(e(f \circ u)), \quad f \mapsto e(T(b) f \circ u)
$$

are bounded linear operators on $L^{2}(\mathbb{T})$. This proves $(2.3)$ for all $b \in L^{\infty}(\mathbb{T})$ and all $f \in H^{2}(\mathbb{T})$.

Now let $\left\{e_{k}\right\}_{k=1}^{\infty}$ be an orthonormal basis in the infinite-dimensional space $K_{u}$.

\section{Lemma 2.4 We have}

$$
H^{2}(\mathbb{R})=\bigoplus_{k=1}^{\infty} E_{u}^{(k)} \quad \text { with } \quad E_{u}^{(k)}:=\left\{e_{k}(f \circ u): f \in H^{2}(\mathbb{T})\right\} .
$$

Proof. If $k_{1} \neq k_{2}$ and $f, g \in H^{2}(\mathbb{T})$, then

$$
\begin{aligned}
\left(e_{k_{1}}(f \circ u), e_{k_{2}}(g \circ u)\right) & =\left(e_{k_{1}} \sum_{j} f_{j} u^{j}, e_{k_{2}} \sum_{l} g_{l} u^{l}\right) \\
& =\sum_{j, l} f_{j} \bar{g}_{l}\left(e_{k_{1}} u^{j}, e_{k_{2}} u^{l}\right) \\
& =\sum_{j} f_{j} \bar{g}_{j}\left(e_{k_{1}} u^{j}, e_{k_{2}} u^{j}\right) \quad(\text { Lee } \\
& =\sum_{j} f_{j} \bar{g}_{j}\left(e_{k 1}, e_{k_{2}}\right)=0 .
\end{aligned}
$$

It remains to prove that $\oplus_{k=1}^{\infty} E_{u}^{(k)}$ is dense in $H^{2}(\mathbb{R})$. Fix an arbitrary $\varepsilon>0$. By Lemma 2.1, there are $h_{1}, \ldots, h_{N} \in K_{u}$ such that

$$
\left\|f-\sum_{j=1}^{N} u^{j} h_{j}\right\|_{2}<\varepsilon .
$$

Each $h_{j}$ can be written as $h_{j}=\sum_{k=1}^{\infty} c_{j k} e_{k}$ with $\sum_{k=1}^{\infty}\left|c_{j k}\right|^{2}<\infty$. Put $g_{k}=\sum_{j=1}^{N} c_{j k} \chi_{j}$. Then $g \circ u=\sum_{j=1}^{N} c_{j k} u^{j}$ and

$$
\left\|f-\sum_{k=1}^{\infty} e_{k}(g \circ u)\right\|_{2}=\left\|f-\sum_{j=1}^{N} u^{j} h_{j}\right\|_{2}<\varepsilon .
$$

This proves that $\oplus_{k=1}^{\infty} E_{u}^{(k)}$ is dense in $H^{2}(\mathbb{R})$. 
We now join the pieces of the puzzle. Let $\mathcal{B}_{L^{\infty}(\mathbb{T})}^{0}(u)$ be the collection of all operators of the form

$$
\sum_{j} \prod_{l} T\left(b_{j l} \circ u\right), \quad b_{j l} \in L^{\infty}(\mathbb{T})
$$

the sum and the product finite, and let $\mathcal{B}_{L^{\infty}(\mathbb{T})}(u)$ denote the closure of $\mathcal{B}_{L^{\infty}(\mathbb{T})}^{0}(u)$ in $\mathcal{L}\left(H^{2}(\mathbb{R})\right)$. Lemma 2.4 gives us the decomposition

$$
H^{2}(\mathbb{R})=\bigoplus_{k=1}^{\infty} E_{u}^{(k)}
$$

and Lemma 2.2(b) tells us that

$$
U_{k}: H^{2}(\mathbb{T}) \rightarrow E_{u}^{(k)}, \quad f \mapsto e_{k}(f \circ u)
$$

is an isometric isomorphism for each $k$. The following theorem clearly implies Theorem 1.1.

Theorem 2.5 Let $u \in H^{\infty}(\mathbb{R})$ be an inner function and suppose $u$ is not a finite Blaschke product. Then the map

$$
F_{u}: \mathcal{B}_{L^{\infty}(\mathbb{T})} \rightarrow \mathcal{L}\left(H^{2}(\mathbb{R})\right), \quad B \mapsto \bigoplus_{k=1}^{\infty} U_{k} B U_{k}^{-1}
$$

is an isometric $C^{*}$-algebra homomorphism satisfying

$$
F_{u}\left(\sum_{j} \prod_{l} T\left(b_{j k}\right)\right)=\sum_{j} \prod_{l} T\left(b_{j k} \circ u\right)
$$

for $\sum_{j} \prod_{l} T\left(b_{j k}\right) \in \mathcal{B}_{L^{\infty}(\mathbb{T})}^{0}$. The image of $F_{u}$ is $\mathcal{B}_{L^{\infty}(\mathbb{T})}(u)$.

Proof. It is clear that $F_{u}$ is an isometric $C^{*}$-algebra homomorphism. From Lemma 2.3 we know that if $b \in L^{\infty}(\mathbb{T})$, then each $E_{u}^{(k)}$ is an invariant subspace of $T(b \circ u)$ and that, for $e_{k}(f \circ u) \in E_{u}^{(k)}$,

$$
T(b \circ u)\left(e_{k}(f \circ u)\right)=e_{k}(T(b) f \circ u)=U_{k} T(b) f=U_{k} T(b) U_{k}^{-1}\left(e_{k}(f \circ u)\right) .
$$

Thus, $T(b \circ u) \mid E_{u}^{(k)}=U_{k} T(b) U_{k}^{-1}$ for each $k$. By virtue of Lemma 2.4 we therefore have

$$
T(b \circ u)=\bigoplus_{k=1}^{\infty} U_{k} T(b) U_{k}^{-1}
$$

which implies that

$$
\sum_{j} \prod_{l} T\left(b_{j l} \circ u\right)=\bigoplus_{k=1}^{\infty} U_{k} \sum_{j} \prod_{l} T\left(b_{j l}\right) U_{k}^{-1}
$$

for every finite collection $\left\{b_{j l}\right\}$ of functions in $L^{\infty}(\mathbb{T})$. This proves $(2.5)$ and shows that the image of $F_{u}$ is $\mathcal{B}_{L^{\infty}(\mathbb{T})}(u)$. 
Theorem 2.6 Let $u \in H^{\infty}(\mathbb{R})$ be an inner function, suppose $u$ is not a finite Blaschke product, let $A \in \mathcal{B}_{L^{\infty}(\mathbb{T})}(u)$, and define $B \in \mathcal{B}_{L^{\infty}(\mathbb{T})}$ by $A=F_{u}(B)$. Then the three equivalences of Theorem 1.2 are valid.

Proof. An operator $T \in \mathcal{L}(H)$ is normally solvable if and only if there exists a number $d>0$ such that $\operatorname{sp}\left(T^{*} T\right) \subset\{0\} \cup\left[d^{2}, \infty\right)$, where sp $(\cdot)$ denotes the spectrum (see, e.g., [8, Theorem 4.21]). From Theorem 2.5 we infer that

$$
\operatorname{sp}\left(F_{u}(B)^{*} F_{u}(B)\right)=\operatorname{sp}\left(F_{u}\left(B^{*} B\right)\right)=\operatorname{sp}\left(B^{*} B\right),
$$

and hence $F_{u}(B)$ is normally solvable if and only if so is $B$.

If $B$ is (left/right) invertible, then, again by Theorem 2.5, $F_{u}(B)$ has the same property. Suppose now that $F_{u}(B)$ is left Fredholm. Then $F_{u}(B)$ is normally solvable and $\operatorname{dim} \operatorname{Ker} F_{u}(B)<\infty$. We already know that $B$ is also normally solvable. If $B f=0$ for some nonzero $f \in H^{2}(\mathbb{T})$, then $F_{u}(B) U_{k} f=0$ for all $k$ due to Theorem 2.5. As the functions $U_{k} f$ are nonzero and pairwise orthogonal, if follows that $\operatorname{dim} \operatorname{Ker} F_{u}(B)=\infty$. This contradiction shows that $\operatorname{Ker} B=\{0\}$, and since $B$ has closed range, we arrive at the conclusion that $B$ is left invertible. Consideration of adjoints now implies that $B$ is right invertible whenever $F_{u}(B)$ is right Fredholm.

Theorems 1.1 and 1.2 follow from Theorems 2.5 and 2.6 with $u=e^{i \alpha}$.

We finally consider the case where $u$ is a finite Blaschke product:

$$
u(x)=\gamma \prod_{j=1}^{n} \frac{x-z_{j}}{x-\bar{z}_{j}}, \quad z_{j} \in \mathbb{C}_{+}, \quad \gamma \in \mathbb{T} .
$$

We then can repeat all the above arguments almost literally, the only difference being that $\operatorname{dim} K_{u}=n<\infty$. It results that $H=\oplus_{k=1}^{n} E_{u}^{(k)}$ and that (2.4) is an isometric isomorphism for every orthonormal basis $\left\{e_{k}\right\}_{k=1}^{n}$ of $K_{u}$. The map

$$
F_{B_{n}}: \mathcal{B}_{L^{\infty}(\mathbb{T})} \rightarrow \mathcal{B}_{L^{\infty}(\mathbb{T})}(u), \quad B \mapsto \bigoplus_{k=1}^{n} U_{k} B U_{k}^{-1}
$$

is a $C^{*}$-algebra isomorphism satisfying (2.5). If $A$ is in $\mathcal{B}_{L^{\infty}(\mathbb{T})}(u)$ and $B \in \mathcal{B}_{L^{\infty}(\mathbb{T})}$ is given by $A=F_{u}(B)$, then

$A$ is normally solvable $\Longleftrightarrow B$ is normally solvable,

$A$ is (left/right) Fredholm $\Longleftrightarrow B$ is (left/right) Fredholm,

$A$ is (left/right) invertible $\Longleftrightarrow B$ is (left/right) invertible,

and if $A$ is left or right Fredholm, then

$\operatorname{dim} \operatorname{Ker} A=n \operatorname{dim} \operatorname{Ker} B, \quad \operatorname{dim}$ Coker $A=n \operatorname{dim}$ Coker $B$. 


\section{The basic algebra homomorphism}

In this section we prove Theorem 1.3.

Thus, suppose $\alpha: \mathbb{R} \rightarrow \mathbb{R}$ is a homeomorphism that preserves the orientation of $\mathbb{R}$ and that satisfies (1.2). In [21], it was shown that (1.2) holds whenever $\alpha$ is twice continuously differentiable and subject to the following conditions

$$
\begin{aligned}
& \liminf _{x \rightarrow+\infty} \frac{x \alpha^{\prime \prime}(x)}{\alpha^{\prime}(x)}>-2, \\
& \lim _{x \rightarrow+\infty} \frac{\alpha^{\prime \prime}(x)}{\left(\alpha^{\prime}(x)\right)^{2}}=0, \\
& \lim _{x \rightarrow+\infty} \frac{x^{1 / 2} \alpha^{\prime \prime}(x)}{\left(\alpha^{\prime}(x)\right)^{3 / 2}}=0, \\
& \lim _{x \rightarrow+\infty}(\alpha(x)+\alpha(-x))=0 .
\end{aligned}
$$

These conditions can be straightforwardly verified if, for example, (3.1) is required a priorily and for large $x>0, \alpha(x)$ is one of the functions

$$
\begin{aligned}
& \alpha(x)=c x^{\gamma}, \quad \gamma>0, \\
& \alpha(x)=c \log ^{\delta} x, \quad \delta>1, \\
& \alpha(x)=c x^{\gamma} \log ^{\delta} x, \quad \gamma>0, \quad \delta>1, \\
& \alpha(x)=c_{1} \exp \left(c_{2} x^{\gamma}\right), \quad \gamma>0, \\
& \alpha(x)=c_{1} \exp \left(c_{2} \exp \left(c_{3} x^{\gamma}\right)\right), \quad \gamma>0,
\end{aligned}
$$

where $c, c_{1}, c_{2}, c_{3}$ are positive constants.

The function $e^{i \alpha}$ can be written in the form

$$
e^{i \alpha(x)}=u_{\alpha}(x) c(x)
$$

where $u_{\alpha}$ is an inner function, $c \in C(\dot{\mathbb{R}})$, and $c(\infty)=1$ (see [6] and [25] for a simple way of reducing the proof to the well known factorization theorems of [28] and [32]). The function $u_{\alpha}$ can in turn be factored into a Blaschke product and a singular inner function. Since $u_{\alpha}$ is continuous on $\mathbb{R}$, the zeros of the Blaschke product cannot have an accumulation point on $\mathbb{R}$. Thus, we have $u_{\alpha}=B S_{\lambda}$ where $B$ is a Blaschke that is continuously differentiable (even $C^{\infty}$ ) on $\mathbb{R}$ and $S_{\lambda}(x)=e^{i \lambda x}$ with $\lambda \geq 0$ (see, e.g., [15] or [25] for details). Clearly, $u_{\alpha}(x)=e^{i \varphi(x)}$ with some real-valued continuously differentiable function $\varphi$ on $\mathbb{R}$. If $\lambda=0$, then $B$ must be an infinite Blaschke product, which implies that $\varphi^{\prime}(x)>0$ for all $x \in \mathbb{R}$. If $\lambda>0$, then $B$ may be a constant or a nonconstant finite or infinite Blaschke product; in any 
case, $\varphi^{\prime}(x) \geq \lambda>0$ for all $x \in \mathbb{R}$. Thus, for each $\gamma \in[0,2 \pi)$ the equation $e^{i \varphi(x)}=e^{i \gamma}$ has a countable number of solutions $\left\{x_{k}(\gamma)\right\}_{k=-\infty}^{\infty}$, which can be labeled so that $3 \leq x_{1}(0) \leq x_{1}(\gamma)<x_{2}(0)$ for all $\gamma \in[0,2 \pi)$ and $x_{k}(\gamma)<x_{k+1}(\gamma)$ for all $k$. Obviously, $x_{k}(\gamma) \rightarrow+\infty$ as $k \rightarrow+\infty$. For $\gamma \in[0,2 \pi)$ and $k \in \mathbb{Z}$, we put

$$
e_{k}^{\gamma}(x)=\mu_{k}^{\gamma} \frac{u_{\alpha}(x)-e^{i \gamma}}{x-x_{k}(\gamma)}, \quad \mu_{k}^{\gamma}:=\frac{1}{\left(2 \pi \varphi^{\prime}\left(x_{k}(\gamma)\right)\right)^{1 / 2}} .
$$

Lemma 3.1 We have $e_{k}^{\gamma} \in K_{u_{\alpha}}$ and $\left\|e_{k}^{\gamma}\right\|_{2}=1$.

Proof. The function $e_{k}^{\gamma}$ can be analytically continued to $\mathbb{C}_{+} \cup D_{\varrho}\left(x_{k}(\gamma)\right)$, where $D_{\varrho}\left(x_{k}(\gamma)\right)$ is the disk of radius $\varrho$ centered at $x_{k}(\gamma)$ and $\varrho>0$ is chosen small enough. Since $\left|e_{k}^{\gamma}(z)\right|$ is bounded for $z \in D_{\varrho}\left(x_{k}(\gamma)\right)$ and decays as $1 /|z|$ as $|z| \rightarrow \infty$ and $\operatorname{Im} z \geq 0$, it follows that

$$
\sup _{y \geq 0} \int_{\mathbb{R}}\left|e_{k}^{\gamma}(x+i y)\right|^{2} d x<\infty .
$$

This proves that $e_{k}^{\gamma} \in H^{2}(\mathbb{R})$ (see, e.g., [15] or [25]). Furthermore, $T\left(\bar{u}_{\alpha}\right) e_{k}^{\gamma}=$ $P g$ with

$$
g(z)=\left(1-\overline{u_{\alpha}(\bar{z})} e^{i \gamma}\right) /\left(z-x_{k}(\gamma)\right)
$$

for $\operatorname{Im} z \leq 0$. In the same way we showed that $e_{k}^{\gamma} \in H^{2}(\mathbb{R})$, we see that $g$ is in the orthogonal complement of $H^{2}(\mathbb{R})$ in $L^{2}(\mathbb{R})$. Thus $P g=0$, and $(2.2)$ implies that $e_{k}^{\gamma} \in K_{u_{\alpha}}$. To compute the norm of $e_{k}^{\gamma}$, note first that

$$
\begin{aligned}
\left\|e_{k}^{\gamma}\right\|_{2}^{2} & =\left(\mu_{k}^{\gamma}\right)^{2} \int_{\mathbb{R}} \frac{2-u_{\alpha}(x) e^{-i \gamma}-\overline{u_{\alpha}(x)} e^{i \gamma}}{\left(x-x_{k}(\gamma)\right)^{2}} d x \\
& =\left(\mu_{k}^{\gamma}\right)^{2} \int_{\Gamma_{\varrho}} \frac{2-u_{\alpha}(z) e^{-i \gamma}-\overline{u_{\alpha}(\bar{z})} e^{i \gamma}}{\left(z-x_{k}(\gamma)\right)^{2}} d z
\end{aligned}
$$

where $\Gamma_{\varrho}$ results from $\mathbb{R}$ by replacing the segment $\left|x-x_{k}(\gamma)\right| \leq \varrho / 2$ by the half-circle $\left\{z \in \mathbb{C}: \operatorname{Im} z \leq 0,\left|z-x_{k}(\gamma)\right|=\varrho / 2\right\}$. As $2 /\left(z-x_{k}(\gamma)\right)^{2}$ and $\overline{u_{\alpha}(\bar{z})} /\left(z-x_{k}(\gamma)\right)^{2}$ are analytic in the lower half-plane and vanish at infinity, Cauchy's theorem shows that the integral (3.4) can be reduced to

$$
\left\|e_{k}^{\gamma}\right\|_{2}^{2}=\left(\mu_{k}^{\gamma}\right)^{2} \int_{\Gamma_{\varrho}} \frac{-u_{\alpha}(z) e^{-i \gamma}}{\left(z-x_{k}(\gamma)\right)^{2}} d z
$$

Finally, Cauchy's integral formula for the complex region above $\Gamma_{\varrho}$ yields

$$
\begin{aligned}
\left\|e_{k}^{\gamma}\right\|_{2}^{2} & =\left(\mu_{k}^{\gamma}\right)^{2} 2 \pi i\left(-u_{\alpha}^{\prime}\left(x_{k}(\gamma)\right)\right) e^{-i \gamma} \\
& =\frac{1}{2 \pi \varphi^{\prime}\left(x_{k}(\gamma)\right)} 2 \pi i\left(-i \varphi^{\prime}\left(x_{k}(\gamma)\right) e^{i \varphi\left(x_{k}(\gamma)\right)}\right) e^{-i \gamma}=1
\end{aligned}
$$


Lemma 3.2 For each $\delta>0$ and each $n \in \mathbb{N}$, there exist $\gamma_{n} \in[0,2 \pi)$ and $k_{n} \geq n$ such that

$$
\varphi^{\prime}\left(x_{k_{n}}\left(\gamma_{n}\right)\right)\left(x_{k_{n}}\left(\gamma_{n}\right)\right)^{3 / 2}>1 / \delta
$$

Proof. Assume the contrary: there are $\delta>0$ and $n \in \mathbb{N}$ such that

$$
\varphi^{\prime}\left(x_{k}(\gamma)\right)\left(x_{k}(\gamma)\right)^{3 / 2} \leq 1 / \delta
$$

for all $\gamma \in[0,2 \pi)$ and all $k \geq n$. There is an $x_{0} \geq 1$ such that each number $x \geq x_{0}$ is $x_{k}(\gamma)$ for some $\gamma \in[0,2 \pi)$ and some $k \geq n$. Thus, (3.5) implies that $\varphi^{\prime}(x) x^{3 / 2} \leq 1 / \delta$ for all $x \geq x_{0}$. Integrating this inequality we get

$$
\varphi(x) \leq \varphi\left(x_{0}\right)+\frac{1}{\delta} \int_{x_{0}}^{x} \frac{d \xi}{\xi^{3 / 2}}<\varphi\left(x_{0}\right)+\frac{1}{\delta} \cdot \frac{1}{2},
$$

which is impossible because $\varphi(x) \rightarrow+\infty$ as $x \rightarrow+\infty$.

Theorem 3.3 Let $b_{j l}$ be a finite collection of functions in $C(\mathbb{T})$ and let

$$
A=\sum_{j} \prod_{l} T\left(b_{j l} \circ e^{i \alpha}\right), \quad B=\sum_{j} \prod_{l} T\left(b_{j l}\right) .
$$

Then $\|A\| \geq\|B\|$.

Proof. Fix an arbitrary $\varepsilon>0$. There is an $f \in H^{2}(\mathbb{T})$ such that $\|f\|_{2}=1$ and $\|B f\|_{2} \geq\|B\|-\varepsilon$. For $k \in \mathbb{N}$ and $\gamma \in[0,2 \pi)$, define $e_{k}^{\gamma}$ by (3.3) and put $\psi_{k}^{\gamma}=e_{k}^{\gamma}\left(f \circ u_{\alpha}\right)$. By Lemma 2.2(b) and Lemma 3.1, $\psi_{k}^{\gamma} \in H^{2}(\mathbb{R})$ and $\left\|\psi_{k}^{\gamma}\right\|_{2}=1$. Let $F_{u_{\alpha}}$ be as in Theorem 1.1 (or Theorem 2.5). Thus,

$$
F_{u_{\alpha}}(B)=\sum_{j} \prod_{l} T\left(b_{j l} \circ u_{\alpha}\right)
$$

Lemmas 2.3 and 3.1 imply that

$$
\begin{aligned}
F_{u_{\alpha}}(B) \psi_{k}^{\gamma} & =\sum_{j} \prod_{l} T\left(b_{j l} \circ u_{\alpha}\right)\left(e_{k}^{\gamma}\left(f \circ u_{\alpha}\right)\right) \\
& =e_{k}^{\gamma}\left(\left(\sum_{j} \prod_{l} T\left(b_{j l}\right) f\right) \circ u_{\alpha}\right)=e_{k}^{\gamma}\left(B f \circ u_{\alpha}\right),
\end{aligned}
$$

whence, again by Lemmas 2.2(b) and 3.1,

$$
\left\|F_{u_{\alpha}}(B) \psi_{k}^{\gamma}\right\|_{2}=\|B f\|_{2} \geq\|B\|-\varepsilon \text {. }
$$


660 A. Böttcher, S. M. Grudsky and E. Ramírez de Arellano

Consequently,

$$
\begin{aligned}
\left\|A \psi_{k}^{\gamma}\right\|_{2} & \geq\left\|F_{u_{\alpha}}(B) \psi_{k}^{\gamma}\right\|_{2}-\|\left(A-F_{u_{\alpha}}(B) \psi_{k}^{\gamma} \|_{2}\right. \\
& \geq\|B\|-\varepsilon-\left\|\left(A-F_{u_{\alpha}}(B)\right) \psi_{k}^{\gamma}\right\|_{2} .
\end{aligned}
$$

We claim that there exists a $\delta>0$ such that if we choose $\gamma_{n} \in[0,2 \pi)$ and $k_{n} \geq n$ as in Lemma 3.2, then

$$
\left\|\left(A-F_{u_{\alpha}}(B)\right) \psi_{k_{n}}^{\gamma_{n}}\right\|_{2}<\varepsilon
$$

for all sufficiently large $n$. Clearly, from (3.6) and (3.7) we obtain that $\|A\|>\|B\|-2 \varepsilon$ (recall that $\left\|\psi_{k_{n}}^{\gamma_{n}}\right\|_{2}=1$ ), and as $\varepsilon>0$ is arbitrary, we arrive at the desired inequality $\|A\| \geq\|B\|$.

To prove our claim (3.7), we may without loss of generality assume that

$$
A=\prod_{l=1}^{m} T\left(b_{l} \circ e^{i \alpha}\right), \quad B=\prod_{l=1}^{m} T\left(b_{l}\right) .
$$

Let first $m=1$ and write $A=T\left(b \circ e^{i \alpha}\right), B=T(b)$. Fix $\delta>0$, let $\gamma_{n}$ and $k_{n}$ be as in Lemma 3.2, and abbreviate $x_{k_{n}}\left(\gamma_{n}\right)$ to $x_{n}$. Notice that $x_{n} \geq 3$ for all $n$ and that $x_{n} \rightarrow \infty$ as $n \rightarrow \infty$. We have

$$
\begin{aligned}
\left\|\left(A-F_{u_{\alpha}}(B)\right) \psi_{k_{n}}^{\gamma_{n}}\right\|_{2}^{2} & =\left\|P\left(\left(b \circ e^{i \alpha}-b \circ u_{\alpha}\right) \psi_{k_{n}}^{\gamma_{n}}\right)\right\|_{2}^{2} \\
& \leq\left\|\left(b \circ e^{i \alpha}-b \circ u_{\alpha}\right) \psi_{k_{n}}^{\gamma_{n}}\right\|_{2}^{2} \\
& =\int_{\mathbb{R}}\left|b\left(e^{i \alpha(x)}\right)-b\left(u_{\alpha}(x)\right)\right|^{2}\left|\psi_{k_{n}}^{\gamma_{n}}(x)\right|^{2} d x
\end{aligned}
$$

We split the integral (3.7) into the integral over $|x|<x_{n}^{1 / 4}$ and the integral over $|x|>x_{n}^{1 / 4}$. The integral over $|x|<x_{n}^{1 / 4}$ does not exceed

$$
\begin{aligned}
& \left(2\|b\|_{\infty}\right)^{2} \int_{|x|<x_{n}^{1 / 4}}\left|e_{k_{n}}^{\gamma_{n}}(x)\right|^{2}|x+i|^{2}\left|\frac{f\left(u_{\alpha}(x)\right)}{x+i}\right|^{2} d x \\
& \quad=\frac{4\|b\|_{\infty}^{2}}{2 \pi} \int_{|x|<x_{n}^{1 / 4}} \frac{\left|u_{\alpha}(x)-e^{i \gamma_{n}}\right|^{2}}{\varphi^{\prime}\left(x_{n}\right)}\left|\frac{x+i}{x-x_{n}}\right|^{2}\left|\frac{f\left(u_{\alpha}(x)\right)}{x+i}\right|^{2} d x .
\end{aligned}
$$

Clearly, $\left|u_{\alpha}(x)-e^{i \gamma_{n}}\right|^{2} \leq 4$. If $|x|<x_{n}^{1 / 4}$, then

$$
\begin{aligned}
|x+i| & <x_{n}^{1 / 4}+1<2 x_{n}^{1 / 4} \\
\left|x-x_{n}\right| & >x_{n}-x_{n}^{1 / 4}=x_{n}^{1 / 4}\left(x_{n}^{3 / 4}-1\right)>x_{n}^{1 / 4} \frac{1}{2} x_{n}^{3 / 4}=\frac{1}{2} x_{n},
\end{aligned}
$$

whence $|x+i|^{2} /\left|x-x_{n}\right|^{2}<8 / x_{n}^{3 / 2}$. 
Thus, (3.9) is at most

$$
\begin{gathered}
\frac{4\|b\|_{\infty}^{2}}{2 \pi} \cdot \frac{4}{\varphi^{\prime}\left(x_{n}\right)} \cdot \frac{8}{x_{n}^{3 / 2}} \int_{|x|<x_{n}^{1 / 4}}\left|\frac{f\left(u_{\alpha}(x)\right)}{x+i}\right|^{2} d x \\
<\frac{64\|b\|_{\infty}^{2}}{\pi} \delta \int_{\mathbb{R}}\left|\frac{f\left(u_{\alpha}(x)\right)}{x+i}\right|^{2} d x
\end{gathered}
$$

the last inequality resulting from Lemma 3.2 (recall also the paragraph before Lemma 2.2). We now consider the integral over $|x|>x_{n}^{1 / 4}$. Since

$$
b\left(e^{i \alpha(x)}\right)-b\left(u_{\alpha}(x)\right)=b\left(u_{\alpha}(x) c(x)\right)-b\left(u_{\alpha}(x)\right)
$$

and $b \in C(\mathbb{T}), c \in C(\dot{\mathbb{R}}), c(\infty)=1$, we see that if $n$ is large enough, then

$$
\left|b\left(e^{i \alpha(x)}\right)-b\left(u_{\alpha}(x)\right)\right|<\frac{\varepsilon}{\sqrt{2}} \quad \text { for } \quad|x|>x_{n}^{1 / 4}
$$

and hence the integral over $|x|>x_{n}^{1 / 4}$ is at most

$$
\begin{aligned}
\frac{\varepsilon^{2}}{2} \int_{|x|>x_{n}^{1 / 4}} & \left|e_{k_{n}}^{\gamma_{n}}(x) f\left(u_{\alpha}(x)\right)\right|^{2} d x \\
& \leq \frac{\varepsilon^{2}}{2} \int_{\mathbb{R}}\left|e_{k_{n}}^{\gamma_{n}}(x) f\left(u_{\alpha}(x)\right)\right|^{2} d x=\frac{\varepsilon^{2}}{2}\left\|\psi_{k_{n}}^{\gamma_{n}}\right\|_{2}^{2}=\frac{\varepsilon^{2}}{2} .
\end{aligned}
$$

Thus, by first choosing $\delta>0$ so that (3.10) is less than $\varepsilon^{2} / 2$ and then choosing $n$ so that (3.11) and therefore (3.12) is satisfied, we obtain that (3.8) is smaller than $\varepsilon^{2}$, which gives (3.7).

Now suppose our claim (3.7) is true for $k \leq m$. We prove it for $m+1$. Let

$$
A=\prod_{l=1}^{m+1} T\left(b_{l} \circ e^{i \alpha}\right), \quad B=\prod_{l=1}^{m+1} T\left(b_{l}\right)
$$

fix $\delta>0$, and pick $\gamma_{n}$ and $k_{n}$ as in Lemma 3.2. Also put

$$
c=b_{m+1} \circ e^{i \alpha}-b_{m+1} \circ u_{\alpha} .
$$

Then

$$
\begin{aligned}
A-F_{u_{\alpha}}(B)= & \left(\prod_{l=1}^{m} T\left(b_{l} \circ e^{i \alpha}\right)-\prod_{l=1}^{m} T\left(b_{l} \circ u_{\alpha}\right)\right) T\left(b_{m+1} \circ u_{\alpha}\right) \\
& +\prod_{l=1}^{m} T\left(b_{l} \circ e^{i \alpha}\right) T(c) .
\end{aligned}
$$

The truth of the claim for $k=1$ shows that

$$
\left\|\prod_{l=1}^{m} T\left(b_{l} \circ e^{i \alpha}\right) T(c) \psi_{k_{n}}^{\gamma_{n}}\right\| \leq \prod_{l=1}^{m}\left\|b_{l}\right\|_{\infty}\left\|T(c) \psi_{k_{n}}^{\gamma_{n}}\right\|_{2}<\frac{\varepsilon}{2}
$$

for all sufficiently large $n$ provided $\delta>0$ is sufficiently small. 
Furthermore, by Lemma 2.3,

$$
T\left(b_{m+1} \circ u_{\alpha}\right) \psi_{k_{n}}^{\gamma_{n}}=e_{k_{n}}^{\gamma_{n}}\left(T\left(b_{m+1}\right) f \circ u_{\alpha}\right)=c e_{k_{n}}^{\gamma_{n}}\left(\tilde{f} \circ u_{\alpha}\right)=: c \widetilde{\psi}_{k_{n}}^{\gamma_{n}}
$$

with $c=\left\|T\left(b_{m+1}\right) f\right\|_{2}$ and $\tilde{f}=c^{-1} T\left(b_{m+1}\right) f$. Thus,

$$
\begin{gathered}
\left(\prod_{l=1}^{m} T\left(b_{l} \circ e^{i \alpha}\right)-\prod_{l=1}^{m} T\left(b_{l} \circ u_{\alpha}\right)\right) T\left(b_{m+1} \circ u_{\alpha}\right) \psi_{k_{n}}^{\gamma_{n}} \\
=c\left(\prod_{l=1}^{m} T\left(b_{l} \circ e^{i \alpha}\right)-\prod_{l=1}^{m} T\left(b_{l} \circ u_{\alpha}\right)\right) \widetilde{\psi}_{k_{n}}^{\gamma_{n}},
\end{gathered}
$$

and as $|c| \leq\left\|b_{m+1}\right\|_{\infty}$ and (3.7) was supposed to be true for $m$, we arrive at the conclusion that the norm of (3.13) is smaller than $\varepsilon / 2$ for all $n$ large enough if only $\delta>0$ is chosen sufficiently small. This completes the proof.

Proof of Theorem 1.3. From Theorem 3.3 we deduce that if

$$
\sum_{j} \prod_{l} T\left(b_{j l} \circ e^{i \alpha}\right)=\sum_{m} \prod_{n} T\left(c_{m n} \circ e^{i \alpha}\right)
$$

then

$$
\sum_{j} \prod_{l} T\left(b_{j l}\right)=\sum_{m} \prod_{n} T\left(c_{m n}\right)
$$

Thus, $G_{\alpha}$ is well-defined on $\mathcal{B}_{C(\mathbb{T})}^{0}\left(e^{i \alpha}\right)$. It is obvious that $G_{\alpha}$ preserves scalar multiples, sums, products and adjoints. As $\mathcal{B}_{C(\mathbb{T})}^{0}\left(e^{i \alpha}\right)$ is dense in $\mathcal{B}_{C(\mathbb{T})}\left(e^{i \alpha}\right)$, Theorem 3.3 implies that $G_{\alpha}$ extends to a $C^{*}$-algebra homomorphism of $\mathcal{B}_{C(\mathbb{T})}\left(e^{i \alpha}\right)$ onto $\mathcal{B}_{C(\mathbb{T})}$.

If $e^{i \alpha}$ is an inner function, $e^{i \alpha}=u$, then $G_{\alpha}$ is the inverse of the isomorphism $F_{u}: \mathcal{B}_{C(\mathbb{T})} \rightarrow \mathcal{B}_{C(\mathbb{T})}(u)$ given by Theorem 1.1. However, if $e^{i \alpha}$ is not an inner function, then $G_{\alpha}$ has a nontrivial kernel. Indeed,

$$
G_{\alpha}\left(T\left(e^{i \alpha}\right) T\left(e^{i \alpha}\right)-T\left(e^{2 i \alpha}\right)\right)=T\left(\chi_{1}\right) T\left(\chi_{1}\right)-T\left(\chi_{1}^{2}\right)=0
$$

but $T\left(e^{i \alpha}\right) T\left(e^{i \alpha}\right)-T\left(e^{2 i \alpha}\right)$ is zero if and only if $e^{i \alpha} \in H^{\infty}(\mathbb{R})$ or $e^{-i \alpha} \in H^{\infty}(\mathbb{R})$ (see [23, Problem 195]). By assumption, $e^{i \alpha} \notin H^{\infty}(\mathbb{R})$. If $e^{-i \alpha}$ were in $H^{\infty}(\mathbb{R})$, then $e^{-i \alpha}$ would be an inner function that is continuous on $\mathbb{R}$. But the continuous arguments of such functions increase monotonically, which contradicts the requirement that $\alpha( \pm \infty)= \pm \infty$. 


\section{The structure theorem and Fredholm criteria}

This section contains the proofs of Theorems 1.4 to 1.6.

We write $T \cong S$ for operators $T$ and $S$ if $T-S$ is a compact operator. We will also make repeated use of the well known fact that

$$
T(a) T(b) \cong T(a b)
$$

if $a, b \in L^{\infty}(\mathbb{R})$ and at least one of the functions $a$ and $b$ is continuous (see, e.g., [7], [8] or [17]). Throughout this section we denote by $u_{\alpha}$ and $c$ the functions given by factorization (3.2).

Lemma 4.1 If $\varphi \in C(\dot{\mathbb{R}})$ and $\varphi(\infty)=0$, then

$$
\begin{aligned}
& F_{u_{\alpha}}(B) T(\varphi) \cong T\left(\left((\operatorname{Sym} B) \circ u_{\alpha}\right) \varphi\right), \\
& T(\varphi) F_{u_{\alpha}}(B) \cong T\left(\varphi\left((\operatorname{Sym} B) \circ u_{\alpha}\right)\right)
\end{aligned}
$$

for every $B \in \mathcal{B}_{L^{\infty}(\mathbb{T})}$.

Proof. We only prove (4.1). The proof of (4.2) is analogous.

Since both sides of (4.1) depend continuously on $B$ (recall Theorem 1.1), it is sufficient to verify (4.1) for

$$
B=\prod_{l=1}^{n} T\left(b_{l}\right), \quad b_{l} \in L^{\infty}(\mathbb{T}) .
$$

We have

$$
F_{u_{\alpha}}(B)=\prod_{l=1}^{n} T\left(b_{l} \circ u_{\alpha}\right), \quad(\operatorname{Sym} B) \circ u_{\alpha}=\prod_{l=1}^{n} b_{l} \circ u_{\alpha} .
$$

Clearly,

$$
T\left(b_{n} \circ u_{\alpha}\right) T(\varphi) \cong T\left(\left(b_{n} \circ u_{\alpha}\right) \varphi\right) .
$$

As $\varphi(\infty)=0$, it follows that $\left(b_{n} \circ u_{\alpha}\right) \varphi$ is in $C(\dot{\mathbb{R}})$ and vanishes at infinity. Hence

$$
T\left(b_{n-1} \circ u_{\alpha}\right) T\left(\left(b_{n} \circ u_{\alpha}\right) \varphi\right) \cong T\left(\left(b_{n-1} b_{n} \circ u_{\alpha}\right) \varphi\right) .
$$

Continuing in this way we obtain

$$
\prod_{l=1}^{n} T\left(b_{l} \circ u_{\alpha}\right) T(\varphi) \cong T\left(\left(\left(b_{1} \cdots b_{n}\right) \circ u_{\alpha}\right) \varphi\right) .
$$


Proof of Theorem 1.4. By virtue of Theorems 1.1 and 1.3, it suffices to prove (1.3) in the case where

$$
A=\prod_{l=0}^{n} T\left(b_{l} \circ e^{i \alpha}\right), \quad b_{l} \in C(\mathbb{T}) .
$$

In that case

$$
F_{u_{\alpha}}(B)=\prod_{l=0}^{n} T\left(b_{l} \circ u_{\alpha}\right), \quad \operatorname{Sym} A=\prod_{l=0}^{n} b_{l} \circ e^{i \alpha}, \quad \operatorname{Sym} F_{u_{\alpha}}(B)=\prod_{l=0}^{n} b_{l} \circ u_{\alpha} .
$$

For $n=0,(1.3)$ is obviously true with $K=0$. Suppose (1.3) is true for $n-1$. We prove it for $n$. Put

$$
c_{0}=b_{0} \circ e^{i \alpha}-b_{0} \circ u_{\alpha}, \quad b=b_{1} \cdots b_{n} .
$$

By assumption,

$$
\begin{aligned}
& \prod_{l=0}^{n} T\left(b_{l} \circ e^{i \alpha}\right)=T\left(b_{0} \circ e^{i \alpha}\right) \prod_{l=1}^{n} T\left(b_{l} \circ e^{i \alpha}\right) \\
& \cong T\left(b_{0} \circ e^{i \alpha}\right)\left(\prod_{l=1}^{n} T\left(b_{l} \circ u_{\alpha}\right)+T\left(b \circ e^{i \alpha}-b \circ u_{\alpha}\right)\right) \\
& =T\left(b_{0} \circ u_{\alpha}+c_{0}\right) \prod_{l=1}^{n} T\left(b_{l} \circ u_{\alpha}\right)+T\left(b_{0} \circ e^{i \alpha}\right) T\left(b \circ e^{i \alpha}-b \circ u_{\alpha}\right) \\
& (4.3)=\prod_{l=0}^{n} T\left(b_{l} \circ u_{\alpha}\right)+T\left(c_{0}\right) \prod_{l=1}^{n} T\left(b_{l} \circ u_{\alpha}\right)+T\left(b_{0} \circ e^{i \alpha}\right) T\left(b \circ e^{i \alpha}-b \circ u_{\alpha}\right) \text {. }
\end{aligned}
$$

Since $c_{0} \in C(\dot{\mathbb{R}})$ and $c_{0}(\infty)=0$, we deduce from Lemma 4.1 that

$$
\begin{aligned}
T\left(c_{0}\right) & \prod_{l=1}^{n} T\left(b_{l} \circ u_{\alpha}\right) \cong T\left(c_{0} \prod_{l=1}^{n} b_{l} \circ u_{\alpha}\right) \\
& =T\left(c_{0}\left(b \circ u_{\alpha}\right)\right)=T\left(\left(b_{0} \circ e^{i \alpha}\right)\left(b \circ u_{\alpha}\right)-\left(b_{0} b\right) \circ u_{\alpha}\right) .
\end{aligned}
$$

Finally, as $b \circ e^{i \alpha}-b \circ u_{\alpha} \in C(\dot{\mathbb{R}})$,

$$
\begin{aligned}
T\left(b_{0} \circ e^{i \alpha}\right) T\left(b \circ e^{i \alpha}-b \circ u_{\alpha}\right) & \cong T\left(\left(b_{0} \circ e^{i \alpha}\right)\left(b \circ e^{i \alpha}-b \circ u_{\alpha}\right)\right) \\
& =T\left(\left(b_{0} b\right) \circ e^{i \alpha}-\left(b_{0} \circ e^{i \alpha}\right)\left(b \circ u_{\alpha}\right)\right) .
\end{aligned}
$$

Inserting (4.4) and (4.5) in (4.3) we get

$$
\prod_{l=0}^{n} T\left(b_{l} \circ e^{i \alpha}\right) \cong \prod_{l=0}^{n} T\left(b_{l} \circ u_{\alpha}\right)+T\left(\left(b_{0} b\right) \circ e^{i \alpha}-\left(b_{0} b\right) \circ u_{\alpha}\right),
$$

which is desired equality. 
Proof of Theorem 1.5. Suppose $A$ is normally solvable. Since $G_{\alpha}$ is a $C^{*}$-algebra homomorphism (Theorem 1.3 ), we can proceed as in the proof of Theorem 2.6 to conclude that $B$ is also normally solvable.

Put $b=\operatorname{Sym} B$. Then $\operatorname{Sym} A=b \circ e^{i \alpha}$ and $\operatorname{Sym}_{u_{\alpha}}(B)=b \circ u_{\alpha}$. Theorem 1.4 tells us that

$$
A \cong F_{u_{\alpha}}(B)+T\left(b \circ e^{i \alpha}-b \circ u_{\alpha}\right) .
$$

Suppose $B$ is left invertible. As $\mathcal{B}_{C(\mathbb{T})}$ is a $C^{*}$-algebra, $B$ has a left inverse $D$ in $\mathcal{B}_{C(\mathbb{T})}$. With $d:=\operatorname{Sym} D$, we have $d b=1$. By (4.6),

$$
F_{u_{\alpha}}(D) A \cong F_{u_{\alpha}}(D) F_{u_{\alpha}}(B)+F_{u_{\alpha}}(D) T\left(b \circ e^{i \alpha}-b \circ u_{\alpha}\right) .
$$

From Theorem 1.1 we know that $F_{u_{\alpha}}(D) F_{u_{\alpha}}(B)=I$. Since $b \circ e^{i \alpha}-b \circ u_{\alpha}$ belongs to $C(\dot{\mathbb{R}})$ and takes the value zero at infinity, Lemma 4.1 gives

$F_{u_{\alpha}}(D) T\left(b \circ e^{i \alpha}-b \circ u_{\alpha}\right) \cong T\left(\left(d \circ u_{\alpha}\right)\left(b \circ e^{i \alpha}-b \circ u_{\alpha}\right)\right)=T\left(\left(d \circ u_{\alpha}\right)\left(b \circ e^{i \alpha}\right)\right)-I$.

Thus,

$$
F_{u_{\alpha}}(D) A \cong T\left(\left(d \circ u_{\alpha}\right)\left(b \circ e^{i \alpha}\right)\right) .
$$

The function $\left(d \circ u_{\alpha}\right)\left(b \circ e^{i \alpha}\right)$ is in $C(\dot{\mathbb{R}})$ and has no zeros on $\dot{\mathbb{R}}$ (note that it equals 1 at infinity). The operator $T\left(\left(d \circ u_{\alpha}\right)\left(b \circ e^{i \alpha}\right)\right)$ is therefore Fredholm. Let $L$ be any inverse modulo compact operators. Then

$$
L F_{u_{\alpha}}(A) A \cong L T\left(\left(d \circ u_{\alpha}\right)\left(b \circ e^{i \alpha}\right)\right) \cong I,
$$

which proves that $A$ is left Fredholm.

Now let $A$ be left Fredholm. The sum

$$
\mathcal{S}:=\mathcal{B}_{C(\mathbb{T})}\left(e^{i \alpha}\right)+\mathcal{K}\left(H^{2}(\mathbb{R})\right)
$$

is a $C^{*}$-subalgebra of $\mathcal{L}\left(H^{2}(\mathbb{R})\right.$ ) (see, e.g., [14, pp. 17-18]). Since $A$ is left Fredholm, the coset $A+\mathcal{K}\left(H^{2}(\mathbb{R})\right)$ is left invertible in $\mathcal{S} / \mathcal{K}\left(H^{2}(\mathbb{R})\right)$. Thus, there is an $L \in \mathcal{B}_{C(\mathbb{T})}\left(e^{i \alpha}\right)$ such that $L A \cong I$. Put $D=G_{\alpha}(L)$ and $d=\operatorname{Sym} D$. Since $\operatorname{Sym} K=0$ for every compact operator, the equality $L A=I+K$ implies that $\left(d \circ e^{i \alpha}\right)\left(b \circ e^{i \alpha}\right)=1$, whence $d b=1$. From (4.6) and the corresponding representation for $L$ we get

$$
\begin{aligned}
I \cong L A & \cong F_{u_{\alpha}}(D) F_{u_{\alpha}}(B)+F_{u_{\alpha}}(D) T\left(b \circ e^{i \alpha}-b \circ u_{\alpha}\right) \\
& +T\left(d \circ e^{i \alpha}-d \circ u_{\alpha}\right) F_{u_{\alpha}}(B)+T\left(d \circ e^{i \alpha}-d \circ u_{\alpha}\right) T\left(b \circ e^{i \alpha}-b \circ u_{\alpha}\right),
\end{aligned}
$$

and Lemma 4.1 therefore gives

$$
\begin{aligned}
I \cong & F_{u_{\alpha}}(D) F_{u_{\alpha}}(B)+T\left(\left(d \circ u_{\alpha}\right)\left(b \circ e^{i \alpha}-b \circ u_{\alpha}\right)\right) \\
& +T\left(\left(d \circ e^{i \alpha}-d \circ u_{\alpha}\right)\left(b \circ u_{\alpha}\right)\right)+T\left(\left(d \circ e^{i \alpha}-d \circ u_{\alpha}\right)\left(b \circ e^{i \alpha}-b \circ u_{\alpha}\right)\right) \\
= & F_{u_{\alpha}}(D) F_{u_{\alpha}}(B)+T\left(\left(d \circ e^{i \alpha}\right)\left(b \circ e^{i \alpha}\right)-\left(d \circ u_{\alpha}\right)\left(b \circ u_{\alpha}\right)\right) \\
= & F_{u_{\alpha}}(D) F_{u_{\alpha}}(B) .
\end{aligned}
$$


Consequently, $F_{u_{\alpha}}(B)$ is left Fredholm. Theorem 1.2 now implies that $B$ is left invertible.

At this point we have proved that $A$ is left Fredholm if and only if $B$ is left invertible. Passage to adjoints shows that $A$ is right Fredholm if and only if $B$ is right Fredholm.

Let us apply Theorem 1.5 to pure Toeplitz operators. We denote by $G C(\mathbb{T})$ the set of all functions in $C(\mathbb{T})$ that have no zeros on $\mathbb{T}$. For $b \in$ $G C(\mathbb{T})$, wind $b$ is the winding number of $b$ about the origin.

Corollary 4.2 Let $b \in C(\mathbb{T})$, let $\alpha: \mathbb{R} \rightarrow \mathbb{R}$ be an orientation preserving homeomorphism satisfying (1.2), and put $a=b \circ e^{i \alpha}$. Then

(a) $T(a)$ is normally solvable if and only if $b$ vanishes identically or $b \in$ $G C(\mathbb{T})$;

(b) if $b \in G C(\mathbb{T})$ and wind $b>0$, then $T(a)$ is left invertible and $\operatorname{dim}$ Coker $T(a)=\infty$;

(c) if $b \in G C(\mathbb{T})$ and wind $b<0$, then $T(a)$ is right invertible and $\operatorname{dim} \operatorname{Ker} T(a)=\infty$;

(d) if $b \in G C(\mathbb{T})$ and wind $b=0$, then $T(a)$ is invertible.

Proof. Let $b \in G C(\mathbb{T})$ and wind $b>0$. Then $T(b)$ is left invertible (see, e.g., [17, Theorem I.7.1]) and hence Theorem 1.5 shows that $T(a)$ is left Fredholm. This implies that $T(a)$ is normally solvable and that $\operatorname{dim} \operatorname{Ker} T(a)<\infty$. If $T(a)$ had a finite-dimensional cokernel, then $T(a)$ would be Fredholm and hence $T(b)$ would be invertible (again Theorem 1.5). But this is not the case if wind $b>0$. Consequently, $\operatorname{dim}$ Coker $T(a)=\infty$. As a nonzero Toeplitz operator always has a trivial kernel or a dense range (Coburn's theorem, see, e.g., [7, Theorem 2.38]), it follows that $\operatorname{dim} \operatorname{Ker} T(a)=\{0\}$. The proof of (b) is complete.

Assertion (c) follows from (b) by passage to adjoints.

Now suppose $b \in G C(\mathbb{T})$ and wind $b=0$. Then $T(a)$ is Fredholm due to Theorem 1.5. The function $b$ is homotopic in $G C(\mathbb{T})$ to the function which is identically 1 . Consequently, $T(a)$ is homotopic in the Fredholm operators to the operator $T\left(1 \circ e^{i \alpha}\right)=I$. Hence $T(a)$ is Fredholm of index zero, which implies that $T(a)$ is invertible (by Coburn's theorem). This completes the proof of $(d)$.

If $T(a)$ is normally solvable, then, by Theorem $1.5, T(b)$ is normally solvable, which in turn happens if and only if $b=0$ or $b \in G C(\mathbb{T})$. Conversely, if $b=0$, then $T(a)=0$ is normally solvable, and if $b \in G C(\mathbb{T})$, then the normal solvability of $T(a)$ can be deduced from (a), (b), (c). The proof of (a) is also complete. 
Proof of Theorem 1.6. For $a \in L^{\infty}(\mathbb{R})$, the Hankel operator $H(a)$ is defined on $H^{2}(\mathbb{R})$ by $H(a) f=P(a(I-P) J f)$, where $(J f)(x):=f(-x)$. Hartman's theorem (see, e.g., [7] or [25]) says that $H(a)$ is compact if and only if $a \in C(\dot{\mathbb{R}})+H_{-}^{\infty}(\mathbb{R})$, where $H_{-}^{\infty}(\mathbb{R})$ stands for the functions in $L^{\infty}(\mathbb{R})$ that can be continued to bounded analytic functions in the lower half-plane $\mathbb{C}_{-}$. Kronecker's theorem (again see, e.g., [7] or [25]) tells us that $H(a)$ has finite rank if and only if $a \in \mathcal{R}+H_{-}^{\infty}(\mathbb{R})$, where $\mathcal{R}$ denotes the set of all bounded rational functions on $\mathbb{R}$. The adjoint operator to $H(a)$ is given by

$$
[H(a)]^{*}=H\left(a^{\#}\right) \quad \text { with } \quad a^{\#}(x):=\overline{a(-x)} .
$$

Finally, for $a$ and $b$ in $L^{\infty}(\mathbb{R})$ we have

$$
T(a b)-T(a) T(b)=H(a) H(J b),
$$

where, as above, $(J b)(x)=b(-x)($ see $[31])$.

Suppose first that $e^{i \alpha} \in C(\dot{\mathbb{R}})+H^{\infty}(\mathbb{R})$ but $e^{i \alpha} \notin \mathcal{R}+H^{\infty}(\mathbb{R})$. Put

$$
A=I-T\left(e^{-i \alpha}\right) T\left(e^{i \alpha}\right) .
$$

Then $B=G_{\alpha}(A)=I-T\left(\chi_{-1}\right) T\left(\chi_{1}\right)=0$, that is, $B$ is normally solvable. From (4.7) and (4.8) we infer that

$$
A=H\left(e^{-i \alpha}\right) H\left(J e^{i \alpha}\right)=K^{*} K \quad \text { with } \quad K=H\left(J e^{i \alpha}\right) .
$$

By Kronecker's theorem, $\operatorname{dim} \operatorname{Im} K=\infty$. Let $e_{1}, e_{2}, \ldots$ be linearly independent elements of $\operatorname{Im} K$. Since $\operatorname{Ker} K^{*} \perp \operatorname{Im} K$ and hence Ker $K^{*} \cap$ $\operatorname{Im} K=\{0\}$, it follows that the elements $K^{*} e_{1}, K^{*} e_{2}, \ldots$ are also linearly independent. Consequently, $\operatorname{dim} \operatorname{Im} K^{*} K=\infty$. But $K^{*}$ and thus $K^{*} K$ are compact due to Hartman's theorem. As compact operators with an infinitedimensional image are never normally solvable, we arrive at the assertion.

Now suppose that $e^{i \alpha} \in \mathcal{R}+H^{\infty}(\mathbb{R})$ but $e^{i \alpha} \notin H^{\infty}(\mathbb{R})$. Pick any irrational number $\mu \in(0,1)$ and let $\zeta^{\mu}$ be the branch of the function which is analytic in $\mathbb{C} \backslash(-\infty, 0]$ and takes the value 1 at $\zeta=1$. The function $b$ defined by $b(t)=(1-t / 2)^{\mu}(t \in \mathbb{T})$ belongs to $H^{\infty}(\mathbb{T})$ and is invertible in $H^{\infty}(\mathbb{T})$. Moreover, both $b$ and $b^{-1}$ have absolutely convergent Fourier series. We consider the operator

$$
A=I-T\left(b \circ e^{i \alpha}\right) T\left(b^{-1} \circ e^{i \alpha}\right) .
$$

Again $B=G_{\alpha}(A)=I-T(b) T\left(b^{-1}\right)=0$ is normally solvable. The assertion will follow once we have shown that $A$ is a compact operator with an infinitedimensional image. By (4.8),

$$
A=H\left(b \circ e^{i \alpha}\right) H\left(J\left(b^{-1} \circ e^{i \alpha}\right)\right) .
$$


Since

$$
\left(b^{-1} \circ e^{i \alpha}\right)(x)=\sum_{n=0}^{\infty}\left(b^{-1}\right)_{n}\left(e^{i \alpha(x)}\right)^{n} \quad \text { and } \quad \sum_{n=0}^{\infty}\left|\left(b^{-1}\right)_{n}\right|<\infty,
$$

we see that $b^{-1} \circ e^{i \alpha} \in C(\dot{\mathbb{R}})+H^{\infty}(\mathbb{R})$. Hartman's theorem therefore implies that $H\left(J\left(b^{-1} \circ e^{i \alpha}\right)\right)$ and thus $A$ is compact.

As $e^{i \alpha} \in \mathcal{R}+H^{\infty}(\mathbb{R})$, we can write $e^{i \alpha}=r+q$ with $r \in \mathcal{R}$ and $q \in H^{\infty}(\mathbb{R})$. Let $z_{1}, \ldots, z_{n}$ be the poles of $r$ in $\mathbb{C}_{+}$, repeated according to their multiplicities. Since $e^{i \alpha} \notin H^{\infty}(\mathbb{R})$, we have $n \geq 1$. The function $r$ can be continued analytically to $\mathbb{C}_{+} \backslash\left\{z_{1}, \ldots, z_{n}\right\}$, and $q$ can be continued analytically to all of $\mathbb{C}_{+}$. Hence, $e^{i \alpha}$ can be continued to some function $F$ analytic in $\mathbb{C}_{+} \backslash\left\{z_{1}, \ldots, z_{n}\right\}$. The point $z_{1}$ is a pole of $F$, which implies that $\left(b^{-1} \circ F\right)(z)=(1-F(z) / 2)^{-\mu}$ is not analytic in a punctured disk of the form $0<\left|z-z_{1}\right|<\varepsilon$. Consequently, $b^{-1} \circ e^{i \alpha}$ cannot be in $\mathcal{R}+H^{\infty}(\mathbb{R})$. This in conjunction with Kronecker's theorem shows that

$$
\operatorname{dim} \operatorname{Im} H\left(J\left(b^{-1} \circ e^{i \alpha}\right)\right)=\infty .
$$

We now prove that

$$
\operatorname{Ker} H\left(b \circ e^{i \alpha}\right)=\{0\} \text {. }
$$

Let $H\left(b \circ e^{i \alpha}\right) h=0$ with some nonzero $h \in H^{2}(\mathbb{R})$ and put $h_{-}=J h$. Then $h_{-}$belongs to $H_{-}^{2}(\mathbb{R})$, the orthogonal complement of $H^{2}(\mathbb{R})$ in $L^{2}(\mathbb{R})$. By the definition of $H\left(b \circ e^{i \alpha}\right)$, there is a function $g_{-} \in H_{-}^{2}(\mathbb{R})$ such that

$$
b\left(e^{i \alpha(x)}\right) h_{-}(x)=g_{-}(x)
$$

for almost all $x \in \mathbb{R}$. Put

$$
B(z)=\prod_{j=1}^{n} \frac{z-z_{j}}{z-\overline{z_{j}}}
$$

Then $B e^{i \alpha}=u$ with some function $u$ that is analytic and bounded in $\mathbb{C}_{+}$ and of modulus 1 on the real line. Thus, $u \in H^{\infty}(\mathbb{R})$ is an inner function. Moreover, $u$ is continuous on $\mathbb{R}$. Let $w_{1}, w_{2}, \ldots$ be the zeros of $u$ in $\mathbb{C}_{+}$. Then $u$ can be continued analytically to $\mathbb{C} \backslash\left\{\overline{w_{1}}, \overline{w_{2}}, \ldots\right\}$ (see, e.g., [15]). As $B^{-1}, h_{-}, g_{-}$can be continued analytically to $\mathbb{C}_{-}$, we deduce from (4.12) that

$$
b\left(u(z) B^{-1}(z)\right) h_{-}(z)=g_{-}(z)
$$

for all $z \in \mathbb{C}_{-} \backslash\left\{\overline{w_{1}}, \overline{w_{2}}, \ldots\right\}$. At the point $z=\overline{w_{k}}$, the function $u(z)$ has a pole. This implies that the left-hand side of (4.13) is not analytic in a neighborhood of $z_{k}$ unless $h_{-}(z)=0$ for all $z$ in a neighborhood of $z_{k}$. 
As $h_{-}$is not identically zero by assumption, we arrive at the conclusion that in fact $\left\{\overline{w_{1}}, \overline{w_{2}}, \ldots\right\}=\emptyset$. Thus, $u$ is a singular inner function of the form $u(x)=\gamma e^{i \lambda x}$ with $|\gamma|=1$ and $\lambda>0$ (the case $\lambda=0$ is ruled out by the requirement that $\alpha$ be monotonically increasing). If $z=x-i y$ with $x \in \mathbb{R}$ and large $y \in(0, \infty)$, then $u(z) B^{-1}(z)$ is close to $\gamma e^{i \lambda x} e^{\lambda y}$, and hence, as $x$ moves from $-\infty$ to $+\infty, u(z) B^{-1}(z)$ necessarily meets the cut $[2, \infty)$ along which $b(z)=(1-z / 2))^{\mu}$ is not analytic. Thus, again we conclude from the analyticity of the left-hand side of (4.13) in $\mathbb{C}_{-}$that $h_{-}$must vanish identically. We have proved (4.11) completely.

Combining (4.10) and (4.11) we see that the operator (4.9) has an infinitedimensional image. This completes the proof.

\section{References}

[1] Barría, J. and Halmos, P. R.: Asymptotic Toeplitz operators. Trans. Amer. Math. Soc. 273 (1982), 621-630.

[2] Beals, R. and Coifman, R.: Scattering and inverse scattering for first order systems. Comm. Pure Appl. Math. 37 (1984), 39-90.

[3] Böttcher, A. And Grudsky, S. M.: Toeplitz operators with discontinuous symbols: phenomena beyond piecewise continuity. In Singular Integral Operators and Related Topics (Tel Aviv, 1995), 55-118. Operator Theory: Advances and Applications 90. Birkhäuser, Basel, 1996.

[4] Böttcher, A. And Grudsky, S. M.: On the composition of Muckenhoupt weights and inner functions. J. London Math. Soc. (2) 58 (1998), $172-184$.

[5] Böttcher, A., Grudsky, S. M. And Spitkovsky, I.: Toeplitz operators with frequency modulated semi-almost periodic symbols. J. Fourier Anal. Appl. 7 (2001), 523-535.

[6] Böttcher, A., Grudsky, S. M. and Spitkovsky, I.: Block Toeplitz operators with frequency-modulated semi-almost periodic symbols. Int. $J$. Math. Math. Sci. 34 (2003), 2157-2176.

[7] Böttcher, A. and Silbermann, B.: Analysis of Toeplitz Operators. Springer-Verlag, Berlin, Heidelberg, New York 1990.

[8] Böttcher, A. And Silbermann, B.: Introduction to Large Truncated Toeplitz Matrices. Universitext, Springer-Verlag, New York 1999.

[9] Coburn, L. A.: The $C^{*}$-algebra generated by an isometry. Bull. Amer. Math. Soc. 73 (1967), 722-726.

[10] Deift, P.: Orthogonal Polynomials and Random Matrices: a RiemannHilbert Approach. Courant Lecture Notes in Mathematics 3. American Mathematical Society, Providence, RI, 1999.

[11] Douglas, R. G.: Banach Algebra Techniques in Operator Theory. Academic Press, New York, 1972 and Springer-Verlag, New York, 1998. 
[12] Douglas, R. G.: Banach Algebra Techniques in the Theory of Toeplitz Operators. CBMS Regional Conference Series in Mathematics 15. American Mathematical Society, Providence, RI, 1973.

[13] Dybin, V.B. And Grudsky, S. M.: Introduction to the Theory of Toeplitz Operators with Infinite Index. Operator Theory: Advances and Applications 137. Birkhäuser Verlag, Basel, 2002.

[14] Fillmore, P. A.: A User's Guide to Operator Algebras. Canadian Mathematical Society Series of Monographs and Advanced Texts. John Wiley \& Sons, Inc., New York, 1996.

[15] Garnett, J. B.: Bounded Analytic Functions. Pure and Applied Mathematics 96. Academic Press, New York-London, 1981.

[16] Gohberg, I.: On an application of the theory of normed rings to singular integral equations. (Russian). Uspehi Matem. Nauk (N.S.) 7 (1952), 149156.

[17] Gohberg, I. and Feldman, I. A.: Convolution Equations and Projection Methods for their Solution. Translations of Mathematical Monographs 41. American Mathematical Society, Providence, RI, 1974.

[18] Gohberg, I. and Krupnik, N.: The algebra generated by the Toeplitz matrices. Funct. Anal. Prilozh. 3 (1969), 119-127.

[19] Gohberg, I. and Krupnik, N.: One-Dimensional Linear Singular Integral Equations. I and II. Operator Theory: Advances and Applications 53 and 54. Birkhäuser Verlag, Basel, 1992.

[20] Grudsky, S. M.: Singular integral operators with infinite index and Blaschke products. (Russian). Math. Nachr. 129 (1986), 313-331.

[21] Grudsky, S. M.: Toeplitz operators and the modelling of oscillating discontinuities with the help of Blaschke products. In Problems and Methods in Mathematical Physics (Chemnitz, 1999), 162-193. Operator Theory: Advances and Applications 121. Birkhäuser, Basel, 2001.

[22] Grudsky, S. M., And Khevelev, A. B.: On the invertibility of singular integral operators with periodic coefficients and a shift in $L^{2}(\mathbb{R})$. Soviet Math. Dokl. 18 (1977), 1383-1387.

[23] Halmos, P.: A Hilbert Space Problem Book. Van Nostrand, Princeton, 1967.

[24] Its, A. R. And Novokshenov, V. Yu.: The Isomonodromic Deformation Method in the Theory of Painlevé Equations. Lecture Notes in Mathematics 1191. Springer-Verlag, Berlin and Heidelberg, 1986.

[25] Nikolski, N. K.: Treatise on the Shift Operator. Grundlehren der Mathematischen Wissenschaften 273. Springer-Verlag, Berlin, 1986.

[26] Nordgren, E. A.: Composition operators. Canad. J. Math. 20 (1968), $442-449$.

[27] Roch, S. And Silbermann, B.: Toeplitz-like operators, quasicommutator ideals, numerical analysis I. Math. Nachr. 120 (1985), 141-173. 
[28] Sarason, D.: Algebras of functions on the unit circle. Bull. Amer. Math. Soc. 79 (1973), 286-299.

[29] Sarason, D.: Toeplitz operators with piecewise quasicontinuous symbols. Indiana Univ. Math. J. 26 (1977), 817-838.

[30] Shapiro, J. H.: Composition Operators and Classical Function Theory. Universitext: Tracts in Mathematics. Springer-Verlag, New York, 1993.

[31] Widom, H.: Asymptotic behavior of block Toeplitz matrices and determinants II. Advances in Math. 21 (1976), 1-29.

[32] Wolff, T. H.: Two algebras of bounded functions. Duke Math. J. 49 (1982), 321-328.

Recibido: 17 de abril de 2002

Albrecht Böttcher
Fakultät für Mathematik
TU Chemnitz
09107 Chemnitz, Germany
aboettch@mathematik.tu-chemnitz.de
Sergei M. Grudsky
Departamento de Matemáticas
CINVESTAV del I.P.N.
Apartado Postal 14-740
07000 México D.F., México
grudsky@math.cinvestav.mx
Enrique Ramírez de Arellano
Departamento de Matemáticas
CINVESTAV del I.P.N.
Apartado Postal 14-740
07000 México D.F., México
eramirez@math. cinvestav.mx

The second author was supported by CONACYT grant, Cátedra Patrimonial, Nivel II, No. 010286 (México). The third author acknowledges partial financial support by CONACYT grant 32726-E (México). 